\title{
Wavelength Conversion by Cavity-Enhanced Injection-Locked Four-Wave Mixing in a Fiber-Bragg-Grating Coupled Diode Laser
}

\author{
Roberto Paiella, Student Member, IEEE, Guido Hunziker, Mehrdad Ziari, Member, IEEE, \\ Atul Mathur, Member, IEEE, and Kerry J. Vahala, Member, IEEE
}

\begin{abstract}
Four-wave mixing (FWM) in a fiber-Bragg-grating (FBG) coupled semiconductor laser is investigated. We show that a large resonance enhancement of the FWM conversion efficiency can be obtained when the laser cavity is injection-locked by the converted signal, and apply this technique to the wavelength conversion of 1-Gb/s modulated signals. Furthermore, we discuss how the spectral width of these resonances can be increased to make this approach suitable to higher bit rates.
\end{abstract}

Index Terms-Communication systems, injection locked oscillators, optical frequency conversion, optical mixing, semiconductor lasers.

A N IMPORTANT phenomenon associated with the optical nonlinearities of semiconductor gain media is fourwave mixing (FWM) between different wavelength channels [1]-[3]. In particular, FWM in semiconductor optical amplifiers (SOA's) is an interesting candidate for wavelength conversion [4]-[6]. In order to optimize the performance of FWM-based devices, much effort has been devoted in the past few years to increasing the FWM conversion efficiency and signal-to-noise ratio (SNR). In this letter, we propose and demonstrate a novel FWM configuration, in which the converted signal frequency is chosen to coincide with a mode of a fiber-Bragg-grating (FBG) coupled diode laser. Through a mechanism similar to injection locking, this results in a large resonance enhancement of both conversion efficiency and SNR. In the device used in this demonstration, this approach is limited to signals with bit rate less than $1 \mathrm{~Gb} / \mathrm{s}$ due to small locking range. However, we show theoretically how the maximum bit rate can be improved by proper design of the laser cavity. A wavelength converter based on this mechanism would then be extremely attractive for applications where tunability of the converted signal frequency is not required. Furthermore, in certain applications, such as selective conversion of one out of several wavelength-division-multiplexing (WDM) channels (e.g., for dynamic channel copying and dropping), the frequency selectivity of this device can actually become an advantage.

Manuscript received December 30, 1997; revised February 6, 1998. This work was supported by ARPA under Contract DAAL 01-94-K-03430 and by the National Science Foundation under Grant ECS-9412862.

R. Paiella, G. Hunziker, and K. J. Vahala are with the Department of Applied Physics, California Institute of Technology, Pasadena, CA 91125 USA.

M. Ziari and A. Mathur are with SDL, San Jose, CA 95134 USA.

Publisher Item Identifier S 1041-1135(98)03823-3.

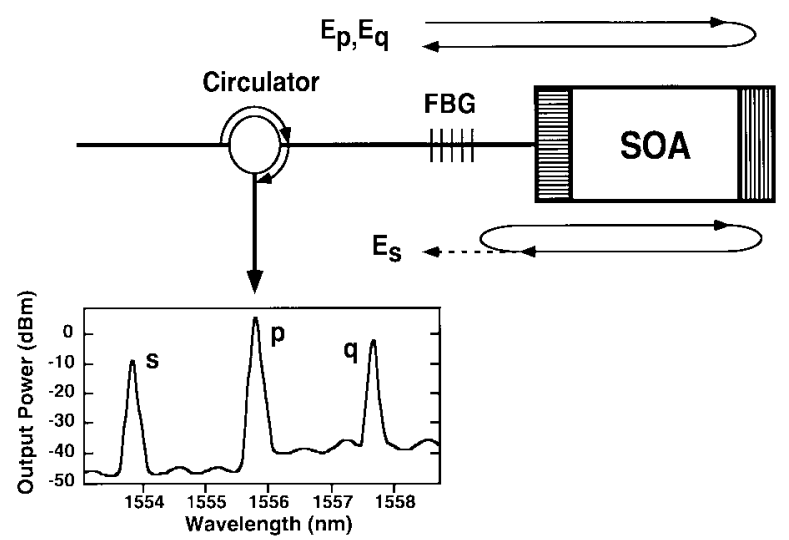

Fig. 1. Schematic representation of the wavelength conversion device studied in this paper. The bottom panel shows the optical spectrum at the output of the converter at resonance $(0.1-\mathrm{nm}$ resolution bandwidth, less than $4 \mathrm{dBm}$ total in-fiber input power).

The approach is based on the external-cavity semiconductor laser shown schematically in Fig. 1, where the optical feedback is provided on one end of the diode by a highreflection coating, and on the other end (antireflection coated) by a pigtailed FBG. This same device was recently used (in a different configuration) for wavelength conversion by selfpumped FWM [7]. In the present experiment, both the input signal and the FWM pump wave are injected into the laser active region through the FBG, using an optical circulator. Their frequencies, $\omega_{q}$ and $\omega_{p}$, respectively, lie well outside of the FBG reflection band, so that for these waves the device simply acts as a (folded-path) SOA. On the other hand, these frequencies are chosen so that the corresponding FWM signal frequency $\omega_{s}=2 \omega_{p}-\omega_{q}$ lies within the reflection band and is closely tuned to a resonance frequency of the laser cavity. The device is biased below the threshold required for laser oscillation in the presence of the pump wave alone. However, when the input signal is turned on, the resulting FWM signal acts as a strong seed for oscillation of the nearby cavity mode, and it is correspondingly enhanced by the lasing action.

We model this enhancement by solving the coupled-mode wave equations for FWM [1], [3] subject to the boundary conditions appropriate to the present configuration (which are illustrated pictorially in Fig. 1). The conversion efficiency $\eta$ (defined as the ratio of the in-fiber converted-signal output power to the input-signal power coupled to the circulator) was 
found to be given by

$$
\frac{\eta}{\left.\eta\right|_{R_{B G} \rightarrow 0}}=\frac{1-R_{B G}\left(\omega_{s}\right)}{\left|1-C \sqrt{R_{B G}\left(\omega_{s}\right)} G\left(\omega_{s}\right) e^{i \Delta \Phi\left(\omega_{s}\right)}\right|^{2}}
$$

where $R_{B G}$ is the power reflectivity of the Bragg mirror, $C$ is the fraction of light that is coupled from the fiber into the SOA (and vice versa), and $G$ and $\Delta \Phi$ are the net gain and phase shift, respectively, experienced by the field amplitude in a round trip in the SOA. The reflectivity of the high-reflection coated facet is taken to be unity. Furthermore, $\left.\eta\right|_{R_{B G} \rightarrow 0}$ denotes the FWM conversion efficiency of the same device under identical gain conditions but without the FBG, so that (1) is a measure of the performance enhancement associated with the optical feedback. In the case of standard laser operation, this same factor gives the ratio of the output power of the laser to the amount of spontaneous-emission noise power emitted into the lasing mode. At threshold, where the gain nearly equals the losses, and for $\omega_{s}$ close to a resonance frequency of the laser cavity, it becomes very large, resulting, in the present case, in a strong resonance enhancement of the FWM conversion efficiency (and SNR). We emphasize the similarity between this mechanism and the technique of injection locking [8] (recently considered for several all-optical switching applications [9], [10]), except that in this case the "master" laser beam is not directly injected, but rather is generated inside the "slave" laser cavity by the FWM interaction between the input waves.

A typical optical spectrum at the output of the device under resonance conditions is shown in Fig. 1, for a wavelength downshift of $4 \mathrm{~nm}$ and an in-fiber input pump power of less than $4 \mathrm{dBm}$. The experimental setup was similar to that described in [7]. Both FWM conversion efficiency and SNR are enhanced by more than two orders of magnitude (relative to the case of FWM in a single-pass, but otherwise identical SOA under the same conditions). In fact, taking into account the amplification experienced by the input signal in the folded-path SOA, the conversion efficiency is close to unity in this case (near threshold the fiber-to-fiber gain is approximately equal to $1 / R_{B G} \approx 6 \mathrm{~dB}$ ). The measured variation of the conversion efficiency with the FWM signal frequency is illustrated in Fig. 2, where several resonances, associated with several modes of the external cavity, are clearly seen. The spectral width of these resonances (the locking range) is found to be quite small, less than $2 \mathrm{GHz}$ for all the peaks in the figure. This poses a limitation to the applicability of the present device to wavelength conversion of high-speed signals, because of the spectral distortion that occurs when the bit rate is larger than the locking range. In a preliminary study, we obtained conversion with best-case BER of $10^{-9}$ at $1 \mathrm{~Gb} / \mathrm{s}$, with eye-diagram as shown in the inset of Fig. 2 (the corresponding BER versus received-power curves exhibit a strong flooring). Also, we found that the conversion performance rapidly degraded with increasing bit rate.

In order to see how the bandwidth of the injection-locking resonances can be increased, it is convenient to recast the conversion efficiency enhancement of (1) in terms of directly mea-

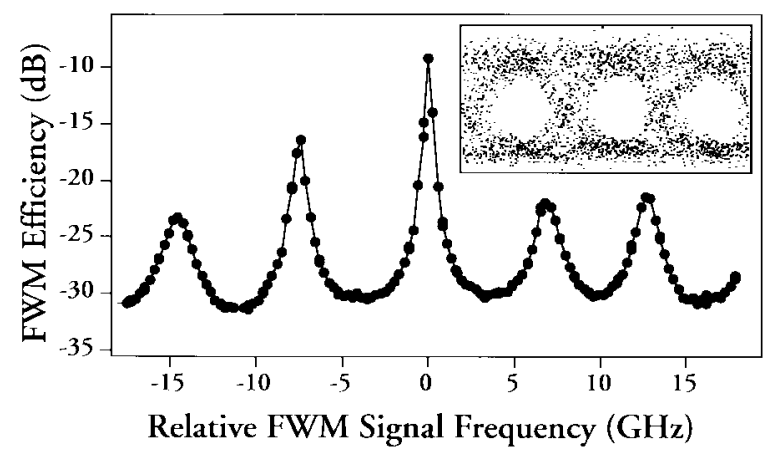

Fig. 2. FWM conversion efficiency versus frequency of the converted signal measured relative to the frequency of the lasing mode (4-nm wavelength down conversion, $\approx 5 \mathrm{dBm}$ total in-fiber input power) and (inset) eye diagram at $1-\mathrm{Gb} / \mathrm{s}$ bit rate.

surable parameters. For $\omega_{s}$ close to any resonance frequency (as will be assumed from here on), (1) is well approximated by a Fabry-Perot transmission peak with FWHM $\delta \nu_{L}$ (a measure of the locking range) and maximum value $E_{\max }$ (the peak enhancement in the FWM conversion efficiency) given by

$$
\begin{aligned}
\delta \nu_{L} & \approx \frac{\Delta \nu}{\pi} \frac{1-e^{\Gamma A \Delta N L}}{e^{\Gamma A \Delta N L / 2}} \\
E_{\max } & \approx \frac{1-R_{B G}\left(\nu_{\mathrm{res}}\right)}{\left(1-e^{\Gamma A \Delta N L}\right)^{2}}
\end{aligned}
$$

where $A$ is the material differential gain, $\Gamma$ is the confinement factor, $L$ is the SOA length, $\nu_{\text {res }}$ is the resonance frequency under consideration, and $\Delta \nu \equiv c /\left[l_{B G}\left(\nu_{\text {res }}\right)+2 n L\right]$ is the cold-cavity free-spectral range (FSR) near $\nu_{\text {res }}\left(l_{B G}\right.$ denotes the effective optical path length from the antireflection (AR)coated facet of the SOA into the Bragg mirror and back to the same facet). Finally, $\Delta N(<0$ under injection-locking conditions) is the deviation of the spatially averaged steadystate carrier density from its value at threshold, given by (for simplicity, here we neglect the longitudinal power variations)

$$
\Delta N=\frac{\left(I-I_{\mathrm{th}}\right) /\left(q V_{a}\right)-g_{\mathrm{th}} \bar{P} /\left(\Gamma \hbar \omega S_{m}\right)}{1 / \tau_{\mathrm{sp}}+A \bar{P} /\left(\hbar \omega S_{m}\right)}
$$

where $I$ is the laser bias current, $V_{a}$ is the volume of the active region, $S_{m}$ is the modal cross-sectional area, $\tau_{\mathrm{sp}}$ is the spontaneous and nonradiative recombination lifetime, $g_{\text {th }}$ is the modal gain coefficient at threshold for the mode under consideration $\left(g_{\mathrm{th}}=\gamma+1 / L \log \left[1 /\left(C \sqrt{R_{B G}\left(\nu_{\text {res }}\right)}\right)\right], \gamma\right.$ being the SOA scattering loss coefficient), and $\bar{P}$ is the spatially averaged steady-state optical power in the $\mathrm{SOA}^{1}$ (which can be easily related to the in-fiber total input power $P^{\text {in }}$ using the FWM coupled-mode equations).

Even though the above analysis strictly applies only to steady-state (i.e., continuous-wave (CW) input waves), the quantity $\delta \nu_{L}$ defined above gives a measure of the maximum

\footnotetext{
${ }^{1}$ Notice that since the main contribution to $\bar{P}$ comes from the $\mathrm{CW}$ pump wave, the entire locking range given above is stable against small carrier density fluctuations (whereas, as originally pointed out by Lang [8] in the context of standard injection locking, if the laser gain is saturated by the injection-locked beam itself, a fraction of the steady-state locking range will actually become unstable for nonzero linewidth enhancement factor).
} 


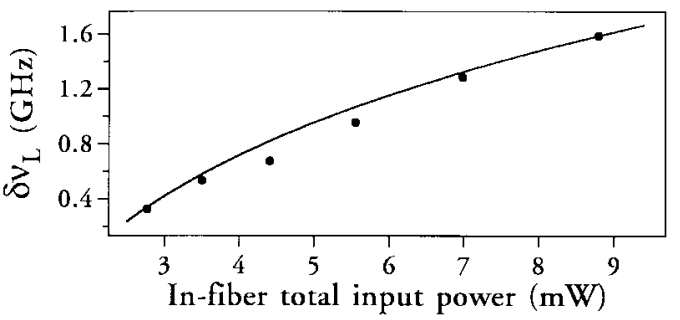

(a)

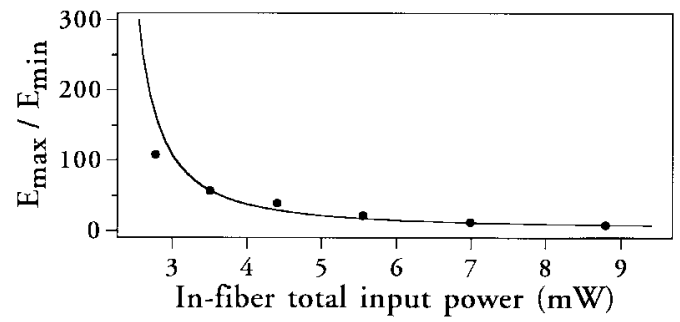

(b)

Fig. 3. Locking range (upper panel) and peak-to-valley ratio of the locking resonance centered at the frequency of the lasing mode (bottom panel) versus total in-fiber input power (4-nm wavelength down conversion, $80-\mathrm{mA}$ bias current). The continuous lines are theoretical fits to the model discussed in the text.

bit rate at which this wavelength converter can operate. Any SOA design effort for this application (the present device was optimized for single-mode laser oscillation) should therefore focus on maximizing this quantity. In fact, this steady-state analysis remains approximately valid even in the presence of a modulated input signal, as long as this is much weaker than the pump (typically the case in FWM wavelength conversion), so that the intensity modulation dynamics is not transferred to the carrier density.

A first observation that follows from (2) is that the width of the locking resonances increases with increasing $\Gamma A|\Delta N| L$; the FWM signal enhancement, however, correspondingly undergoes a (larger) decrease. To illustrate this point, we plot in Fig. $3 \delta \nu_{L}$ and $E_{\max } / E_{\min }$ (the peak-to-valley ratio of the resonance, given by $\left.\left[\left(1+e^{\Gamma A \Delta N L}\right) /\left(1-e^{\Gamma A \Delta N L}\right)\right]^{2}\right)$ versus input power $P^{\text {in }}\left[|\Delta N|\right.$ increases with $\bar{P}$, and hence with $P^{\text {in }}$, as confirmed by (3)]. The circles in the figure are experimental values (corresponding to the resonance peak centered at the lasing frequency); the continuous lines are theoretical fits based on the expressions above. The quantity $\Gamma A|\Delta N| L$ should therefore be optimized to achieve the largest possible $\delta \nu_{L}$ for which the FWM conversion efficiency remains larger than a given threshold (e.g., large enough to support error-free wavelength conversion in a given link). As clearly indicated by Fig. 3, this can be simply accomplished by varying the input pump power.
To make further progress, the maximum bit rate can be increased by increasing the FSR $\Delta \nu$ (in particular by minimizing the optical path length in the fiber $l_{B G}$, which, in the present device, accounts for most of the measured 7-GHz FSR), since $\delta \nu_{L}$ increases linearly with it, while the conversion efficiency is not affected. In practice this means, for instance, writing the grating as close to the lensed fiber tip as possible, and reducing its length (the corresponding decrease in reflectivity, while increasing the laser threshold, is if anything advantageous for this application since it increases $E_{\max }$ ). In particular, based on the present experimental results, if $\Delta \nu$ could be increased by about a factor of three, the device would become well suited to operation at $2.5 \mathrm{~Gb} / \mathrm{s}$, the prevalent bit rate per channel currently used in telecommunication systems. On the other hand, for operation at $10 \mathrm{~Gb} / \mathrm{s}$ and faster, more dramatic design innovations are likely required, such as, for instance, using an on-chip DBR to provide the optical feedback (this would also make the FWM signal wavelength tunable). In any case, this approach to wavelength conversion is very interesting, in light of its large conversion efficiency and low-input power requirements.

\section{REFERENCES}

[1] G. P. Agrawal, "Population pulsations and nondegenerate four-wave mixing in semiconductor lasers and amplifiers," J. Opt. Soc. Amer. B, vol. 5, pp. 147-158, 1988.

[2] J. Zhou, N. Park, J. W. Dawson, K. J. Vahala, M. A. Newkirk, and B. I. Miller, "Terahertz four-wave mixing spectroscopy for study of ultrafast dynamics in a semiconductor optical amplifier," Appl. Phys. Lett., vol. 63, pp. 1179-1181, 1993.

[3] A. Mecozzi, S. Scotti, A. D’Ottavi, E. Iannone, and P. Spano, "Fourwave mixing in traveling-wave semiconductor amplifiers," IEEE $J$. Quantum Electron., vol. 31, pp. 689-699, 1995.

[4] D. F. Geraghty, R. B. Lee, M. Verdiell, M. Ziari, A. Mathur, and K. J. Vahala, "Wavelength conversion for WDM communication systems using four-wave mixing in semiconductor optical amplifiers," IEEE J. Select. Topics Quantum Electron., vol. 3, pp. 1146-1155, Oct. 1997.

[5] M. A. Summerfield and R. S. Tucker, "Optimization of pump and signal powers for wavelength converters based on four-wave mixing in semiconductor optical amplifiers," IEEE Photon. Technol. Lett., vol. 8, pp. 1316-1318, 1996.

[6] A. D’Ottavi, F. Girardin, L. Graziani, F. Martelli, P. Spano, A. Mecozzi, S. Scotti, R. Dall'Ara, J. Eckner, and G. Guekos, "Four-wave mixing in semiconductor optical amplifiers: A practical tool for wavelength conversion," IEEE J. Select. Topics Quantum Electron., vol. 3, pp. 522-528, 1997.

[7] G. Hunziker, R. Paiella, M. Ziari, A. Mathur, and K. J. Vahala, "Foldedpath self-pumped wavelength converter based on four-wave mixing in a semiconductor optical amplifier," IEEE Photon. Technol. Lett., vol. 9, pp. 1352-1354, 1997.

[8] R. Lang, "Injection locking properties of a semiconductor laser," IEEE J. Quantum Electron., vol. 18, pp. 976-983, 1982.

[9] K. Weich, E. Patzak, and J. Höorer, "Fast all-optical switching using two-section injection-locked semiconductor lasers," Electron. Lett., vol. 30, pp. 493-494, 1994.

[10] L. Li and K. Petermann, "Small-signal analysis of optical-frequency conversion in an injection-locked semiconductor laser," IEEE J. Quantum Electron., vol. 30, pp. 43-48, 1994. 